Meta

Journal des traducteurs

Translators' Journal

\title{
Les nouvelles règles de traduction du Vatican
}

\section{Jean Delisle}

Volume 50, numéro 3, août 2005

Le prisme de l'histoire

The History Lens

URI : https://id.erudit.org/iderudit/011599ar

DOI : https://doi.org/10.7202/011599ar

Aller au sommaire du numéro

Éditeur(s)

Les Presses de l'Université de Montréal

ISSN

0026-0452 (imprimé)

1492-1421 (numérique)

Découvrir la revue

\section{Citer cet article}

Delisle, J. (2005). Les nouvelles règles de traduction du Vatican. Meta, 50(3), 831-850. https://doi.org/10.7202/011599ar

\section{Résumé de l'article}

Après avoir rappelé brièvement l'attitude de l'Église catholique à l'égard des traductions et des traducteurs au cours de son histoire et en particulier aux IV $^{\mathrm{e}}$ et $\mathrm{XVI}^{\mathrm{e}}$ siècles, nous examinons les principaux documents émanant du Vatican depuis 1943 afin de dégager la conception de l'Église à l'égard de la traduction. Nous analysons en détail la cinquième instruction post-conciliaire, Liturgiam authenticam (2001), véritable " traité de traduction " dans lequel Rome édicte des règles précises et contraignantes pour la traduction de la Bible et des textes liturgiques. En conclusion, nous portons un jugement critique sur la vision de la traduction qui se dégage de ce traité.
Ce document est protégé par la loi sur le droit d'auteur. L'utilisation des services d'Érudit (y compris la reproduction) est assujettie à sa politique d'utilisation que vous pouvez consulter en ligne.

https://apropos.erudit.org/fr/usagers/politique-dutilisation/ 


\title{
Les nouvelles règles de traduction du Vatican'
}

\author{
JEAN DELISLE \\ Université d'Ottawa, Ottawa, Canada \\ jdelisle@uottawa.ca
}

\section{RÉSUMÉ}

Après avoir rappelé brièvement l'attitude de l'Église catholique à l'égard des traductions et des traducteurs au cours de son histoire et en particulier aux $I V^{e}$ et $X V I^{e}$ siècles, nous examinons les principaux documents émanant du Vatican depuis 1943 afin de dégager la conception de l'Église à l'égard de la traduction. Nous analysons en détail la cinquième instruction post-conciliaire, Liturgiam authenticam (2001), véritable «traité de traduction » dans lequel Rome édicte des règles précises et contraignantes pour la traduction de la Bible et des textes liturgiques. En conclusion, nous portons un jugement critique sur la vision de la traduction qui se dégage de ce traité.

\begin{abstract}
After a brief look at the attitude of the Catholic Church towards translation and translators throughout history, and particularly in the 4th and 16th centuries, we will examine the key Vatican documents published since 1943, with the intention of showing the Church's notions of translation. Particular attention will be given to the fifth postVatican II instruction, Liturgiam authenticam (2001), which is actually a treatise on translation, in which Rome has laid down precise and stringent rules for translating the Bible and liturgical texts. We will conclude by casting a critical eye on the conceptions (or misconceptions) of translation found in the treatise.
\end{abstract}

\section{MOTS-CLÉS/KEYWORDS}

histoire de la traduction, règles de traduction, Église catholique, Vatican, instructions post-conciliaires

«La Bible a été et demeure l'ambassadrice auprès des nations occidentales de la sagesse orientale. Les ambassadeurs qui restent trop longtemps loin de leur patrie risquent, on le sait, d'en oublier le vrai visage. Ainsi en a-t-il été de la Bible.»

(ANDré Chouraqui, L'amour fort comme la mort, 1990)

\section{Rappel historique}

Depuis ses origines, l'Église catholique a partie liée avec la traduction, à tel point que l'on a pu écrire que «traduire est le mouvement originel du christianisme» (Boyer 2002: 120), que «traduire la Bible c'est toujours traduire de la traduction» (ibid.: $69)$ et que "la traduction de la Bible est une activité typiquement chrétienne ${ }^{2}$ » (Rabin 1972: 16). Les textes fondamentaux du christianisme publiés en langues vernaculaires sont, on le sait, des traductions de traductions... dont l'original n'existe plus. Donc des traductions de copies de copies. On comprend dès lors que l'Église catholique ait toujours fait preuve de vigilance et de prudence, voire d'une certaine méfiance à l'égard des traductions, pourtant indispensables à sa mission: pas d'évangélisation 
sans traduction. On se souvient qu'au $\mathrm{IV}^{\mathrm{e}}$ siècle circulaient dans la chrétienté plusieurs traductions en langues syriaque, arménienne, éthiopienne, grecque et latine, dont la Vetus latina. Cette traduction, la plus ancienne en cette langue, fut réalisée entre 200 et 250 en Afrique proconsulaire par des communautés chrétiennes qui ne comprenaient pas le grec, langue de l’Église primitive. Comme ces versions divergeaient entre elles et renfermaient de nombreuses erreurs, un travail de révision s'imposait. Le pape Damase $\mathrm{I}^{\mathrm{er}}$ (v. 305-384) confia alors à Eusebius Hieronymus (Jérôme, v. 347-420), la redoutable tâche de réviser la Bible. Son travail, réalisé à Bethléem, aboutit à la Vulgate 3 . Le docteur de Stridon était l'homme de la situation; il réunissait toutes les compétences linguistiques et philologiques nécessaires pour accomplir cette révision. À la fois philosophe, rhéteur, grammairien, dialecticien et traducteur, Jérôme avait une connaissance approfondie de l'hébreu, du grec et du latin. Il est non seulement le premier traducteur-réviseur de la Bible, mais aussi le premier véritable théoricien de la traduction. Ses nombreux écrits renferment une conception cohérente de la traduction (Kelly 1975: 162-163; Kelly 1976). Dans les siècles qui ont suivi, l'Église fit de sa Vulgate un livre canonique.

$\mathrm{Au} \mathrm{XVI}^{\mathrm{e}}$ siècle, les réformistes protestants en Europe multiplient les traductions en langues vulgaires pour que les fidèles puissent lire et interpréter la Bible par euxmêmes, sapant par le fait même le monopole de l'interprétation des Écritures que l'Église romaine s'était arrogé ${ }^{4}$ La contre-réforme et l'Inquisition furent les manifestations de la réaction de Rome, qui s'opposait farouchement à cette prolifération de traductions, considérée comme source d'erreurs et de division. Au plus fort de la Réforme ${ }^{5}$, l'Église a jugé nécessaire, lors du concile de Trente (1545-1563) au cours duquel furent examinés tous les points fondamentaux de la doctrine catholique, de proclamer officiellement l'édition de la Vulgate comme la seule version authentique des Saintes Écritures, déclaration qui du coup discréditait aux yeux des catholiques toutes les versions en langues vulgaires et les rendaient nulles et non avenues. Lors de sa séance du 8 avril 1546, le Concile

a considéré qu'il pourrait être d'une grande utilité pour l'Église de Dieu de savoir, parmi toutes les éditions latines des livres saints qui sont en circulation, celle que l'on doit tenir pour authentique: aussi statue-t-il et déclare-t-il que la vieille édition de la Vulgate, approuvée dans l'Église même par un long usage de tant de siècles, doit être tenue pour authentique dans les leçons publiques, les discussions, les prédications et les explications, et que personne n'ait l'audace ou la présomption de la rejeter sous quelque prétexte que ce soit (Denzinger 2001: $\mathrm{n}^{\circ}$ 1506).

L'Église ne toléra que les traductions accompagnées d'annotations fiables tirées des écrits des pères et docteurs de l'Église catholique.

Si elle se montrait méfiante à l'égard des traductions, Rome, jalouse de son privilège exclusif d'interprétation des Écritures, ne voyait pas d'un bon oil non plus que les fidèles, le «commun ignorant» selon l'expression de Pie VII, s'adonnent à la libre lecture des Saintes Écritures. En 1816, ce souverain pontife écrivait: "Si la sainte Bible est admise en langue vulgaire en tous lieux, sans discrimination, il en résulte plus de dommage que d'utilité» (Pie VII 1816). Les traductions en langues vernaculaires étant forcément différentes les unes des autres - il est dans la nature même de la traduction de faire différent -, les chefs de l'Église ont toujours craint que ces multiples traductions «ébranlent l'immutabilité du témoignage divin» et fassent «chanceler la foi» (ibid.). Plus que toute autre institution, l'Église catholique souffre 
de la malédiction de Babel. C'est pourquoi elle a toujours entretenu une relation ambivalente à l'égard de la traduction et a souvent tenu les traducteurs pour «hérétiques». Elle a tendance à les soupçonner de vouloir propager insidieusement des erreurs doctrinales en éditant la Bible dans des langues que les fidèles peuvent lire et comprendre, ce qui a pour effet de saper son pouvoir. Sans être dépositaire des livres originaux (la Bible hébraïque est antérieure au christianisme), l'Église s'estime néanmoins dépositaire de plein droit de l'orthodoxie chrétienne. Aussi traduire la Bible peut-il devenir un acte périlleux, une activité subversive. Les censeurs ecclésiastiques se tiennent aux aguets pour traquer les traducteurs qui osent défier l'autorité romaine en proposant de nouvelles versions des Écritures. Plusieurs de ces traducteurs courageux ont péri sur le bûcher (Dolet, Tyndale, Hus), d'autres ont été emprisonnés (Fray Luis de León), persécutés ou contraints de s'expatrier (Calvin, Lefèvre d'Étaples, Marot, Robert Estienne), déclarés hérétiques et excommuniés (Wyclif, Luther), mis à l'index (Littré, Renan et combien d'autres...), ou encore ont vu leurs écrits subir un autodafé ou être abondamment caviardés (Érasme). Les moyens de répression dont dispose l'Église et dont les traducteurs ont été victimes au cours de l'histoire sont nombreux. «Si un traducteur doit payer son travail de sa vie ou de sa liberté, c'est que les enjeux de son activité sont parfois plus élevés qu'on ne le croit» (Pym 1997: 12).

On peut dire que l'Église catholique n'a jamais été tendre envers les traducteurs, pourtant indispensables à son rayonnement universel. En 1844, le pape Grégoire XVI porte un jugement sévère sur eux et rappelle les dangers que représentent les traductions en langues vulgaires quand elles ne portent pas le sceau de Rome:

Vous n'ignorez pas, écrit-il, quelle diligence et quelle sagesse sont requises pour traduire fidèlement dans notre langue les paroles du Seigneur, puisque aussi bien rien ne se produit plus facilement que ces erreurs très graves introduites dans les traductions multipliées par les sociétés bibliques, et qui proviennent de la sottise et de la tromperie de tant de traducteurs; et ces erreurs, le grand nombre même et la diversité de ces traductions les occultent pendant longtemps au détriment de beaucoup. À ces sociétés elles-mêmes il importe peu ou pas du tout qu'en lisant ces bibles traduites en langue vulgaire les hommes tombent dans telles erreurs plutôt que dans d'autres, pourvu qu'ils s'accoutument peu à peu à revendiquer pour eux- mêmes un libre jugement concernant le sens des Écritures, à mépriser les traditions divines gardées dans l'Église sur la base de la doctrine des Pères, et à rejeter le magistère de l'Église elle-même (Grégoire XVI 1844).

Deux ans plus tard, Pie IX revient à la charge et accuse les «perfides sociétés bibliques» de renouveler «les artifices odieux des anciens hérétiques » et de produire «contre les règles si sages de l'Église, les livres des saintes Écritures traduits en toute espèce de langues vulgaires, et souvent expliquées [sic] dans un sens pervers », et de les répandre parmi les fidèles les moins instruits (Pie IX 1846). Le point de vue exprimé par les trois papes susmentionnés correspond assez bien à l'attitude historique de l'Église à l'égard de la traduction et des traducteurs.

Depuis 1943, le Vatican a été amené, par la force des circonstances, à préciser de façon plus nuancée sa position en ce qui concerne la traduction de la Bible et des textes liturgiques. Un certain nombre de documents officiels émanant de Rome traitent, en effet, de traduction et témoignent de l'importance cruciale que l'Église accorde à cette question: 
1943 Divino Afflante Spiritu (Pie XII) - Lettre encyclique sur les études bibliques

1963 Sacrosanctum Concilium - Constitution conciliaire sur la sainte liturgie

1964 Inter Ecumenici - Première instruction. Principes pour l'application de la constitution sur la liturgie

1967 Tres abhinc annos - Deuxième instruction. Nouvelles adaptations des rites de la messe

1970 Liturgicae instaurationes - Troisième instruction. Directives sur le rôle de l'évêque pour le renouveau liturgique

1979 Nova Vulgata - Promulgation par Jean-Paul II de la Néo-Vulgate (édition typique ${ }^{6}$ latine)

1988 Vicesimus Quintus Annus - Lettre apostolique de Jean-Paul II, publiée à l'occasion du $25^{\mathrm{e}}$ anniversaire de la promulgation de la Constitution Sacrosanctum Concilium

1994 Varietates legitimae - Quatrième instruction. Sur l'inculturation et la liturgie romaine

2001 Liturgiam authenticam - Cinquième instruction. De l'usage des langues vernaculaires dans l'édition des livres de la liturgie romaine

Dans la suite du présent article, nous tâcherons de cerner l'évolution de la pensée de l'Église en matière de traduction au cours des quelque soixante dernières années.

\section{La traduction-inculturation}

Le coup d'envoi est donné en 1943 par Pie XII, qui publie sa lettre encyclique Divino Afflante Spiritu. Ce document marque une évolution par rapport à la position traditionnelle de l'Église, qui s'ouvre davantage aux études bibliques et aux traductions. À propos de la révision des livres sacrés, le pape affirme que s'ils renferment des erreurs, les seuls responsables en sont les copistes (et les traducteurs), car ces livres sont d'inspiration divine et «l'inspiration divine exclut toute erreur» (art. 6). Les études exégétiques peuvent bénéficier des progrès réalisés dans plusieurs sciences auxiliaires, dont l'histoire, l'archéologie, l'ethnologie et la philologie. L'exégète contemporain est ainsi mieux armé pour «chercher à saisir religieusement et avec le plus grand soin les moindres détails sortis de la plume de l'hagiographe sous l'Esprit Divin » (art. 20). Il peut donc s'employer à purifier de ses altérations le «texte primitif écrit par l'auteur sacré lui-même» et à le délivrer des gloses, lacunes, inversions de mots, répétitions et "des fautes de tout genre qui ont coutume de se glisser dans tous les écrits transmis à travers plusieurs siècles» (art. 21). Dans cette encyclique, le pape souhaite également «dissiper [le] préjugé selon lequel l'Église voit d'un mauvais œil et entrave la lecture de l'Écriture ${ }^{7} »$ (art. 13). Il se montre favorable à la publication de nouvelles traductions de la Bible en langues vernaculaires ${ }^{8}$, à condition que ces traductions soient faites à partir des langues « originales ${ }^{9}$ » et de la Vulgate, qui «est absolument exempte de toute erreur en ce qui concerne la foi et les mœurs» (art. 26). Pie XII précise que les traducteurs-exégètes doivent «mettre le plus grand soin à découvrir [le] sens littéral des mots ${ }^{10} »($ art. 27). S'il reste des obscurités, c'est qu'elles sont voulues par l'auteur divin: «Dieu a parsemé à dessein de difficultés les Livres Saints qu'il a inspirés lui-même, afin de nous inciter à les lire et à les scruter avec d'autant plus d'attention et pour nous exercer à l'humilité par la constatation salutaire de la capacité limitée de notre intelligence» (art. 41). Il faut la foi pour croire cela... L'encyclique Divino Afflante Spiritu reconnaît, en somme, que les livres sacrés 
peuvent être révisés et qu'il est possible d'en proposer de nouvelles traductions. Ce n'est pas un hasard si elle fut promulguée un 30 septembre, jour anniversaire de la mort de saint Jérôme, qui a consacré la moitié de sa vie précisément à ce travail.

Le Concile Vatican II (1962-1966) ouvre encore davantage la porte à la traduction. En 1963, Paul VI promulgue la Constitution sur la sainte liturgie, Sacrosanctum Concilium, afin de faciliter l'application du renouveau liturgique souhaité par les pères conciliaires. Dans ce document important, puisqu'il incarne l'esprit de renouveau qui souffle alors sur l'Église, Rome autorise («concède» est le mot employé dans la version française de la Constitution) l'usage d'autres langues que le latin pour la messe, l'administration des sacrements, les prières et autres actes rituels. Toute nouvelle traduction des textes bibliques ou liturgiques doit toutefois recevoir l'aval du Siège apostolique. Depuis la publication de ce document, le Saint-Siège a rendu publiques cinq «Instructions pour la bonne application de la Constitution sur la sainte Liturgie du Concile Vatican II »: Inter Ecumenici (1964), Tres abhinc annos (1967), Liturgicae instaurationes (1970), Varietates legitimae (1994), Liturgiam authenticam (2001). Toutes ces instructions comportent des dispositions concernant la traduction. Les deux dernières portent plus spécifiquement sur la révision des livres liturgiques en langue latine et sur leur traduction dans les différentes langues modernes.

Varietates legitimae traite de la délicate question de l'inculturation. On entend par ce terme la présentation et la réexpression de la bonne parole dans des formes et des termes propres à une culture. L'inculturation est indissociable de la démarche évangélisatrice de l'Église, puisque la culture est un aspect essentiel de la personne humaine. Elle est le prisme par lequel l'être humain fait l'expérience du monde, y compris l'expérience spirituelle. Avec la publication de Sacrosanctum Concilium, Rome accepte que le rite romain s'assouplisse quelque peu et adopte diverses autres formes ${ }^{11}$, à l'intérieur de certaines limites, bien entendu.

Plus intéressant encore, de notre point de vue: l'instruction Liturgiam authenticam, la dernière en date, porte exclusivement sur la traduction en langues vernaculaires. Sans doute pour la première fois de sa longue histoire, Rome y expose en détail, sous forme de règles précises, sa conception de la traduction et ses exigences particulières pour la traduction des textes bibliques et liturgiques. Véritable «traité de traduction", ce document, qui remplace toutes les normes et directives précédentes à l'exception de celles figurant dans Varietates legitimae, pose des balises destinées à encadrer le travail des traducteurs. En fait, il s'agit davantage d'un «règlement», car cet ensemble de prescriptions est coercitif. Ces règles ne sont pas données simplement à titre indicatif. Elles découlent directement de l'expérience acquise au cours des trente dernières années, caractérisées par l'adaptation des textes bibliques et liturgiques dans diverses langues et cultures.

Il importe de préciser que, dans le sillage de l'aggiornamento que les évêques conciliaires appellent de leurs vœux, le successeur de Jean XXIII, Paul VI, crée une commission spéciale dès 1965 et lui confie le mandat de réviser la Vulgate, à la lumière des progrès modernes réalisés par l'érudition biblique ${ }^{12}$. Une version préliminaire de ce qu'il est convenu d'appeler la Nova Vulgata ou Néo-Vulgate paraît en 1970. La version finale est promulguée par Jean-Paul II en 1979, mais le Vatican ne ferme pas pour autant la porte à toute amélioration de ce texte canonique ${ }^{13}$. Édition typique, la Néo-Vulgate ne remplace pas les textes en langues originales, mais constitue un document de référence auxiliaire pour la traduction en langues vernaculaires. Cette édition 
offre l'avantage, selon Rome, de maintenir la tradition en matière d'interprétation des textes fondateurs. Certains livres de la Bible, dont ceux de Samuel, nous ont été transmis en plusieurs versions hébraïques et grecques divergentes. Quelle tradition choisir lorsqu'il faut les traduire? Désormais le traducteur n'aura plus le choix: il lui faudra suivre l'interprétation retenue dans la Néo-Vulgate. Dans ce contexte bien particulier, la traduction sert de point de référence doctrinal.

Depuis Vatican II, on peut dire qu'en matière de traduction Rome n'était pas opposée en principe aux traductions qui appliquaient la technique de l'adaptation et des «équivalences dynamiques", ce qui allait tout à fait dans le sens de l'inculturation et facilitait la compréhension des textes par les fidèles. Le latin, langue officielle des affaires de l'Église, était, hors des officines de la hiérarchie catholique, une langue morte, incomprise de la masse des croyants. Dans la liturgie, après deux mille ans de règne sans partage, le latin cédait enfin la place aux langues vivantes; le célébrant ne tournait plus le dos au peuple, mais lui faisait face. Ce retournement est symbolique.

\section{Le retour du balancier}

Vingt-cinq ans à peine après la promulgation de la Constitution Sacrosanctum Concilium (1963), Jean-Paul II publie en 1988 une lettre apostolique, Vicesimus Quintus Annus, dans laquelle transparaît son inquiétude au sujet de l'évolution des réformes en matière de liturgie et de traduction:

Les Conférences épiscopales ont eu la lourde charge de préparer les traductions des livres liturgiques. Les nécessités du moment ont parfois conduit à utiliser des traductions provisoires, qui ont été approuvées ad interim.

Mais le temps est venu de réfléchir à certaines difficultés éprouvées depuis, de remédier à certaines faiblesses ou inexactitudes, de compléter les traductions partielles, de créer ou d'approuver les chants à utiliser dans la liturgie, de veiller au respect des textes approuvés, de publier enfin des livres liturgiques dans un état qu'on peut considérer comme acquis durablement et dans une présentation qui soit digne des mystères célébrés. Pour le travail de traduction, mais aussi pour une concertation plus large à l'échelle du pays entier, les Conférences épiscopales devaient constituer une Commission nationale et s'assurer le concours de personnes expertes dans les différents secteurs de la science et de l'apostolat liturgique. Il convient de s'interroger sur le bilan, positif ou négatif, de cette Commission, sur les orientations et sur l'aide qu'elle a reçues de la Conférence des évêques dans sa composition ou son activité. Le rôle de cette Commission est beaucoup plus délicat quand la Conférence veut traiter de certaines mesures d'adaptation ou d'inculturation plus profondes: c'est une raison de plus pour veiller à y placer des personnes vraiment expertes (Jean-Paul II 1988, art. 20).

Pourquoi ce temps d'arrêt et de réflexion? Que s'est-il passé en vingt-cinq ans? D’où provient l'inquiétude du souverain pontife? La hiérarchie romaine, dépositaire et gardienne de la Vérité révélée, a-t-elle jugé qu’il y a eu débordement, dérapage dogmatique, non-respect de la tradition? Tout porte à croire que cette manière de traduire qui fait place à une forme de créativité contenue n'a pas produit les résultats escomptés. Certaines initiatives d'inculturation (donc de traduction) ne semblent pas avoir eu l'heur de plaire au Siège apostolique. On peut facilement imaginer que c'est le cas des nouvelles traductions qui associent des spécialistes des Écritures bibliques (pour le fond) et des écrivains contemporains (pour la forme), des traductions où Dieu est présenté sous les traits d'une femme, où la Vierge Marie est de race noire, aux 
traductions en langue basique, familière, argotique ${ }^{14}$, aux versions idéologiques, féministes ${ }^{15}$, poétisées, paraphrasées (Living Bible), aux traductions-calques érudites et autres productions similaires. Cette prolifération quelque peu anarchique de versions «tendancieuses» qui malmènent, aux yeux de l'autorité romaine, la doctrine traditionnelle n'est pas sans rappeler la situation qui régnait au temps de Jérôme et plus encore à l'époque de la Réforme, comme nous l'avons évoqué précédemment. Chaque fois qu'il y a péril en la demeure, Rome réagit. Cette fois, ce n'est pas en créant des tribunaux d'Inquisition ni en érigeant des bûchers qu'elle neutralisera les «traducteurs hérétiques». Sa stratégie consiste à agir de l'intérieur en établissant des critères sévères de sélection des traducteurs, en promulguant des règles coercitives de traduction et en mettant en place des procédures rigoureuses d'approbation des seules traductions sur lesquelles elle peut apposer son estampille d' "authenticité». Rome reprend donc l'initiative et redéfinit unilatéralement les règles du jeu. Une fois de plus la bataille se déplace sur le champ de la traduction, une fois de plus les traducteurs sont dans la mire de l'Église. L'étau se resserre sur eux. Comme sur tous ces croyants de bonne volonté qui, par conviction, récrivent la Bible, en tout ou en partie, dans un langage accessible au «commun ignorant».

\section{La traduction-tradition}

En vingt-cinq ans, l'Église et la société ont connu des mutations profondes et rapides. Le phénomène d'inculturation de même que les traductions en langues vernaculaires qui l'ont accompagné ont soulevé de nouveaux problèmes, imprévus à l'origine. Les nouvelles règles de traduction rendues publiques par le Vatican visent à remédier à la situation.

En général, la traduction supporte mal la camisole de force qu'imposent des règles contraignantes. Le traducteur voudrait-il les appliquer à la lettre que les particularités et subtilités des textes l'en empêcheraient. Il ne peut exercer convenablement son art sans une certaine liberté de réexpression, sans faire appel aux indispensables ressources de la recréation. Traduire n'a rien d'un banal processus de substitution. «Le travail traductif requiert un sujet libre, libre dans son choix fondamental de traduction, libre dans ses choix ponctuels, libre dans la maîtrise de cette chaîne de "coup par coup" (J.-R. Ladmiral) qu'est le traduire dans sa pratique à ras de texte. Cette liberté-là se confond avec la fidélité, et il appartient à chaque traducteur, non sans risque, de délimiter l'espace de jeu de cette liberté-fidèle ${ }^{16}$ » (Berman 1995: 42). C'est pourquoi, de toutes les règles consignées dans les traités et les arts de traduire, bien peu, à vrai dire, ont eu une réelle utilité pratique. Aucun traducteur, à notre connaissance, n'a jamais indiqué avoir appliqué dans son travail l'ensemble des règles d'un traité de traduction. Une règle de traduction ne sera jamais une règle de grammaire; un traité de traduction ne sera jamais un manuel d'instructions. "La traduction est une activité où l'on suit des règles sans disposer de règles pour appliquer les règles", a écrit fort pertinemment Christian Berner (1999: 18). Les règles qui composent ces traités sont énoncées a posteriori et témoignent d'une conception parfois très personnelle de la manière de traduire, quand elles ne codifient pas tout simplement des généralités ou des pratiques établies.

Il en va tout autrement des règles contenues dans l'instruction Liturgiam authenticam, qui porte en sous-titre: «De l'usage des langues vernaculaires dans l'édition 
des livres de la liturgie romaine.» Ces directives sont édictées par une institution puissante, autocratique, qui est en mesure d'imposer d'autorité à ses traducteurs des normes et des pratiques de traduction rigoureuses. Faute de les appliquer, les traducteurs verront leur traduction refusée par le Saint-Siège, qui n'accordera pas son imprimatur ${ }^{17}$. Nous sommes aux antipodes de la liberté créatrice dont jouissent les traducteurs littéraires. De ce point de vue, on aurait tort d'assimiler la traduction religieuse, telle que la conçoit le Vatican, à la traduction littéraire, du seul fait du caractère profondément littéraire des livres qui composent la Bible. Ses règles, comme nous le verrons, faussent la donne. On peut traduire une ouvre littéraire en la privant de sa littérarité. Que vaut, par exemple, un poème dépouillé de son rythme, de sa musicalité, de sa signifiance? C'est un corps sans vie.

Examinons de plus près les «principes qui devront être suivis désormais dans les futures traductions» (art. 71) pour que ces versions puissent être certifiées conformes à la doctrine de l'Église. L'instruction Liturgiam authenticam ${ }^{18}$ comporte cinq grandes subdivisions:

I - Le choix des langues vernaculaires en vue de leur introduction dans la liturgie

II - La traduction de textes liturgiques dans les langues vernaculaires

III - La préparation des traductions et l'établissement des commissions

IV - La publication des livres liturgiques

$\mathrm{V}$ - La traduction des textes liturgiques propres $^{19}$.

L'objet de l'instruction est clairement exposé: «Réfléchir de nouveau sur la notion juste de traduction liturgique, de telle sorte que les traductions de la sainte Liturgie en langue vernaculaire soient d'une manière certaine la voix authentique de l'Église de Dieu» (art. 7).

Le texte original latin renferme une note de bas de page très significative. On y indique que le latin possède plusieurs mots pour désigner l'acte de traduire, notamment versio, conversio, interpretatio, redditio, mutatio, transductio. Ces termes synonymes sont couramment employés dans la Constitution Sacrosanctum Concilium et dans de nombreux autres documents contemporains. Mais les auteurs de l'instruction les écartent d'emblée au profit de translatio et des mots de la même famille, «même si l'usage de ces mots est un peu dur quant au style en latin, et entre dans la catégorie des "néologismes" " (art. 2, note 2). Ce qui peut sembler une banale simplification terminologique est en fait l'indice d'un parti pris théorique sur la manière de traduire. Toutes les autres expressions sont écartées, car elles comportent «la notion d'une divergence ou variation du texte nouveau par rapport au sens du texte original» (ibid.). Elles laissent croire que des modifications porteuses d'éventuelles erreurs doctrinales ont été apportées aux textes. Ainsi, dès le départ, les auteurs annoncent leurs couleurs: ils se déclarent adeptes de la traduction littérale, et entretiennent l'illusion qu'il est possible, en passant d'une langue à une autre, de réaliser des «copies conformes », sans gauchissement, sans changement, sans transformation aucune. Ils conçoivent la translatio plus ou moins comme un acte de «report» non déformant. La réflexion théorique moderne nous a appris que cela est un mythe. Le report, tel que nous avons défini ce concept ${ }^{20}$, porte uniquement sur certains éléments d'un texte de départ et il n'est pas applicable indistinctement à toutes les parties du discours. Le processus de la traduction implique l'interprétation du sens, et la réexpression de ce sens appréhendé se fait soit par le rappel de formules plus ou moins consacrées, données d'avance dans la langue d'arrivée (c'est la «remémoration»), soit par la 
postulation d'une équivalence lexicale, syntagmatique ou même phrastique, imprévisible hors discours (c'est la «création discursive»). Concevoir la traduction comme une opération assimilable à un transvasement garant d'exactitude, c'est avoir une conception réductrice et erronée de cette activité complexe. Les littéralistes purs et durs, les Chateaubriand ${ }^{21}$, Nabokov, Newmark ${ }^{22}$ et autres, manifestent une véritable obsession à l'égard de l'exactitude; leur erreur est de chercher cette exactitude dans les mots, alors que le sens émane du discours. Traduire, ce n'est pas singer, ce n'est pas une opération de clonage de mots. «L'exactitude n'est que du sens. C'est-à-dire seulement une partie du sens. Puisqu'elle oublie que le sens n'a lui-même qu'une part dans le mode de signifier» (Meschonnic 2004: 173). En traduction, et a fortiori en traduction biblique, on ne peut pas faire l'économie de la poétique des textes si on veut réellement les traduire. Une traduction est un acte de langage spécifique. Elle ne peut pas se nier elle-même, se présenter comme si elle n'existait pas et prétendre donner directement à lire le texte qu'elle traduit, comme l'a bien vu Arno Renken: «Un texte bien traduit est un texte qui serait identique à l'original, ce qui n'est pas seulement une impossibilité pratique, mais encore une aberration théorique puisque cela implique une aliénation de la traduction au moment même de sa réalisation » (Renken 2002: 17).

Toutes les langues naturelles du monde se valent en droit et en dignité. Toutes, cependant, ne pèsent pas du même poids quant à leur pouvoir de communication et de diffusion. De ce point de vue, il y a une hiérarchie des langues. L'instruction limite le nombre de celles qu'il est licite d'admettre dans les célébrations liturgiques, donc de traduire. Sont exclues les langues qui «demeurent seulement un objet d'intérêt culturel» (art. 10) et dont les chances de survie sont minces. Il se fera donc dorénavant moins de traduction dans des dialectes ou des parlers locaux voués à disparaître. Les auteurs du document reconnaissent - et l'histoire leur donne raison - que l'introduction, par l'Église, des langues vernaculaires dans la liturgie peut avoir un effet déterminant sur le sort de ces langues. Il n'empêche que le choix des langues dans lesquelles se feront les traductions est un choix idéologique.

Rome réaffirme haut et fort les vertus du littéralisme. Désormais, ce sera au lecteur à faire l'effort de comprendre l'original. Alors que les pères conciliaires prônaient une langue simple, concise, claire et exempte de répétitions inutiles, l'instruction Liturgiam authenticam bannit toute forme de créativité (art. 20) et impose la traduction la plus littérale possible, même si les mots utilisés traditionnellement sont obsolètes, archaïques ou obscurs. Les articles 19 à 69 de l'instruction énoncent en détail les règles et contraintes diverses auxquelles les traducteurs devront se soumettre pour traduire «le texte original ou primitif [...] intégralement et très précisément» (art. 20), sans omissions, ajouts, paraphrases ou gloses. Quelques brefs exemples serviront à illustrer le genre de littéralisme que les auteurs de l'instruction préconisent. À la formule courante de salutation Dominus vobiscum traduite en anglais par The Lord be with you, les fidèles avaient l'habitude de répondre par And also with you. Désormais, il leur faudra dire (comme en français): And with your spirit, car cette formule calque le latin Et cum spirito tuo. Autres exemples: le Credo doit être traduit obligatoirement à la première personne du singulier, et les mots «résurrection de la chair», traduits littéralement (art. 65). Est bannie la formulation: «la résurrection des morts».

On ne veut plus d'un style trop simplifié d'où sont éliminées les subordonnées, les figures de rhétorique et les répétitions. La langue sacrée et solennelle doit maintenant 
reprendre ses droits et se substituer à la langue courante que parle la masse des fidèles. Pourtant, dans l'histoire, la Bible «a été le creuset d'une intense créativité linguistique et littéraire, de jeux de langage rendus nécessaires par la tâche complexe de la transmission des écrits polyphoniques, véritables millefeuilles d'expressions religieuses et culturelles, d'expériences de sens. Vouloir aujourd'hui arrêter cette créativité, dénoncer le travail de "translation", c'est en réalité se faire les embaumeurs hypocrites de ce que l'on prétend admirer et vouloir continuer à transmettre» (Boyer 2002: 41). Une langue qui refuse de se renouveler s'opacifie, se fige, se désincarne. Les mots de la Bible finissent par devenir des coquilles creuses, par ressembler à «d'imprenables forteresses vides» (ibid. : 46) et paraissent, aux yeux de certains, insubstituables, intraduisibles. Fixisme asphyxiant. "Les mots péché, grâce, foi, résurrection ne supporteraient pas d'autres traductions qu'elles-mêmes»(ibid.). Ces mots portent le sceau d'un héritage et d'une tradition, celle de la langue du religieux chrétien.

S'il faut créer des termes nouveaux ou élargir l'aire sémantique de mots déjà en usage dans les langues vernaculaires, ces nouveautés doivent répondre aux vraies exigences culturelles et pastorales et ne pas être introduites simplement pour faire neuf ou différent (art. 21, 22). On retiendra de préférence les termes traditionnels de la Néo-Vulgate, tels que Alleluia, Amen, Kyrie eleison. Seraient rejetées par les censeurs ecclésiastiques des équivalences « revisitées» telles que «éveiller» (Cor. 15,4) au lieu de «ressusciter» (Boyer 2001), «Les Psaumes» rendus par «Gloires», Amen explicité et paraphrasé en «C'est ma foi », "péché » et «pécheur» par «égarement » et "égaré» (Meschonnic 2001); les «anges» renommés "griffons», «messagers» ou «kéroubim» ou encore «Le Nouveau Testament» rebaptisé «Un Pacte neuf» (Chouraqui 1989). Les traducteurs travaillant au service du Vatican doivent s'en tenir le plus possible à la langue chrétienne de transmission des Écritures, au vocabulaire du religieux chrétien traditionnel, tout comme les traducteurs d'une grande entreprise sont tenus d'utiliser la terminologie maison de l'entreprise. De ce point de vue, l'Église catholique, une et universelle, ne diffère guère des grandes multinationales aux ramifications tentaculaires et au sein desquelles l'uniformisation de la terminologie en usage dans les communications écrites et la description des produits et procédés de fabrication sont une préoccupation constante. De toute évidence, en édictant cette cinquième instruction, Rome n'a aucunement l'intention de décaper le langage de la Bible, de le rajeunir, de le nettoyer des couches culturelles qui s'y sont superposées au fil des siècles. Et pourtant, n’y a-t-il pas «à déchristianiser, à déshelléniser, à délatiniser, à débondieuser [...], à défranciser ce qu'on nous donne à lire comme des traductions en français de la Bible» (Meschonnic 2004: 149) ? Il faut «réhébraïser la Bible. En français. Et dans toutes les langues où on la traduit. Où on traduit. Après des siècles à la fois d'édulcoration et d'annexion langagière, mais aussi de perversion idéologique » (ibid. : 151). Réhébraïser, certes, mais en faisant en sorte que le message biblique ne perde pas sa signification ni sa pertinence pour ceux à qui il s'adresse. «Toutes les traductions françaises de la Bible me font mal au cœur, " écrivait Paul Claudel (1966: 13). Mais Rome reste obstinément attachée à la langue du discours chrétien qui s'est formée au cours des siècles et sa dernière instruction ne va aucunement dans le sens d'un renouvellement de cette langue.

Il est désormais interdit de faire des traductions à partir d'autres traductions, ce qui est une règle d'éthique tout à fait valable et qui figure également dans les codes de déontologie des associations de traducteurs professionnels ${ }^{23}$. Compte tenu du contexte 
général dans lequel l'instruction a été élaborée, il est permis de penser que l'objectif non avoué de cette règle vise aussi à réduire le nombre de traductions en circulation. Les traductions des textes liturgiques seront dorénavant effectuées uniquement à partir des versions officielles latines, tandis que les Saintes Écritures seront traduites à partir de l'hébreu, de l'araméen ou du grec, tout en tenant compte, pour les usages liturgiques, de l'incontournable Néo-Vulgate, témoin privilégié de la tradition de l'Église (art. 24).

Les mots employés dans les traductions doivent respecter la dignité et la beauté du contenu doctrinal exact des textes. Ces termes, précise-t-on, doivent être «libres de toute adhésion trop étroite à des modes d'expression du moment» (art. 27). Soigneusement choisis, ils doivent contribuer à la formation d'un «style sacré » de cette langue du religieux chrétien évoquée plus haut: «Une traduction liturgique qui transmet l'autorité et l'intégrité du sens des textes originaux sert à former une langue sacrée vernaculaire, dont le vocabulaire, la syntaxe et la grammaire sont appropriés au culte divin, sans pour autant perdre la force et l'autorité qu'ont ces éléments dans le langage quotidien, comme cela fut le cas dans les langues des peuples d'antique évangélisation» (art. 47). Les expressions obsolètes ou inélégantes en usage dans certains passages de la Bible seront conservées. Cette règle implique, semble-t-il, dans l'esprit de ses auteurs que la stabilité du vocabulaire reflète la stabilité et, partant, la crédibilité de l'institution et de sa doctrine. Il en va de même pour les symboles, les images et les actes rituels qui doivent "parler d'eux-mêmes». On évitera d' "expliciter grossièrement toutes les subtilités des allusions bibliques» (art. 49). Les archaïsmes et les mots vieillis seront préférés aux néologismes au destin incertain et parfois éphémère. Finies donc les traductions rapprochantes, explicatives ou ethnocentriques. La même règle s'applique aux mots concrets qui décrivent les actions des êtres célestes représentés sous des traits humains, tels que marcher, bras, doigt, main, visage de Dieu, chair, corne, bouche, germe, visites, etc. Tous ces mots relevant de l'anthropomorphisme, il est préférable «de ne pas les aplatir, ni de les rendre dans les langues vernaculaires par des termes plus abstraits ou vagues» (art. 43). Toujours ce même culte idolâtre rendu aux mots traditionnels de l'expression de la foi chrétienne. Sacralisation, en fait, de traductions anciennes dont le vocabulaire s'est sédimenté, pétrifié. Glorification d'un état de langue passé. Refus de croire aux possibilités expressives des langues modernes pour faire entendre le message divin. Refus des risques que comporte le travail sur la langue.

Deux articles, 30 et 31, portent sur la délicate question du genre grammatical des noms et des pronoms. Faut-il représenter Dieu comme un homme ou une femme? Le mot homme peut-il désigner le genre humain? Quel est le sexe des anges? Les auteurs de l'instruction tranchent: «[... si le texte originel emploie un mot unique pour exprimer le lien entre un seul homme et l'unité, ainsi que l'universalité de la famille ou la communauté humaine (comme les mots hébreu adam, grec anthropos, latin homo), il faut conserver cette manière de s'exprimer» (art. 30). N'en déplaise aux féministes: le mot homme est inclusif et continuera à désigner l'ensemble des êtres humains. L'article suivant (32) renferme les consignes d'ordre grammatical:

a) Dieu et les membres de la Sainte Trinité sont de sexe masculin, leur genre grammatical étant masculin.

b) La locution composée Filius hominis, en raison de son importance christologique, sera traduite obligatoirement par une locution similaire (Fils de l'homme). 
c) Le mot Patres (Pères) sera toujours rendu par un mot masculin.

d) L'Église sera toujours désignée par un pronom féminin (cf. notre Sainte Mère l'Église).

e) Ce sera le genre grammatical du mot du texte original qui déterminera en langues vernaculaires le genre des anges, des démons, des déesses et des dieux païens.

Cette dernière consigne est une autre preuve de la force d'attraction qu'exercent les textes originaux. Cela s'applique même aux majuscules, dont l'emploi en langue d'arrivée se modèlera sur l'édition typique en langue latine.

Afin d'assurer uniformité et stabilité (art. 36), une seule traduction approuvée sera autorisée sur un même territoire. Il est demandé aussi aux traducteurs d'éviter d'employer un vocabulaire ou un style que les catholiques pourraient confondre avec des manières de s'exprimer d'autres communautés religieuses non catholiques. Le Vatican souhaite également, dans un souci évident de préserver la cohérence, l'uniformité et l'harmonisation de l'ensemble du corpus doctrinal, qu'il y ait une concordance étroite entre les textes des prières, des lectionnaires ${ }^{24}$ et de la liturgie romaine, et le texte de la Néo-Vulgate. Les traducteurs feront en sorte que leur manière de traduire favorise cette correspondance (art. 49), ce qui est une contrainte supplémentaire venant s'ajouter à celle-ci: veiller à ce que «la traduction exprime le sens traditionnel christologique, typologique ou spirituel, et que soient manifestés l'unité et le lien entre l'Ancien et le Nouveau Testament» (art. 41). On demande ni plus ni moins aux traducteurs de «christianiser» l'Ancien Testament en montrant qu'il préfigure le Nouveau. En histoire, cela s'appelle un anachronisme; en sciences sociales, une idéologie.

Ce même article propose une règle d'interprétation lorsqu'un texte donne lieu à des interprétations multiples: le sens du passage difficile à traduire sera élucidé, notamment, à la lumière de la Néo-Vulgate, de la tradition, des écrits des pères de l'Église et, ce qui étonne, des anciennes traductions des Saintes Écritures, dont la Septante ou Bible d'Alexandrie ${ }^{25}$. On sait que la Septante est une mauvaise traductioncalque émaillée d'erreurs grossières et coulée dans un grec très hébraïsé. On peut penser qu'elle figure dans cette règle d'interprétation surtout en raison de son littéralisme excessif qui la rapprocherait des textes primitifs, de cette veritas hebraica que prônait saint Jérôme. En traduction religieuse, plus on est littéral, plus on est, croit-on, près de la "vérité scripturaire», plus on a l'impression d'être le fidèle porte-parole de l'auteur initial. Indéracinable mythe!... quoi qu'en pense le littéraliste bon teint Vladimir Nabokov pour qui «l'expression "traduction littérale" est tautologique, car une traduction qui n'est pas littérale n'est pas une véritable traduction, mais une imitation, une adaptation ou une parodie» (Nabokov 1955: 504; notre traduction). C'est faire bien peu de cas de toute la dimension discursive et poétique des textes. C'est théoriser la traduction en termes de langue et de signifiants en oubliant qu'un texte a aussi une signifiance. Le traducteur n'est pas un eunuque à qui l'on confie la garde des mots.

Les auteurs de Liturgiam authenticam, document dans lequel sont abordés la plupart des aspects fondamentaux de la traduction, n'ont pas jugé bon d'utiliser le métalangage moderne servant à décrire les concepts et les opérations de la traduction. Ils n’emploient aucune expression spécialisée telle que «langue de départ», «langue d'arrivée», " texte cible», «texte source», "procédé de traduction», «stratégie de traduction », «intertextualité», etc. Ils y traitent, néanmoins, de dénotation et de connotation, de l'attitude à adopter à l'égard des termes latins difficiles à traduire, 
des cas où l'emprunt se justifie et où la création néologique est licite, des registres de discours et des multiples niveaux de signification des textes. Les articles 57, 58 et 59 portent sur la syntaxe, les procédés de style et les genres littéraires.

Aux nombreuses contraintes imposées aux traducteurs s'en ajoutent deux autres: «traduire le texte pour qu'il puisse être mis en musique» (art. 60) et tenir compte du temps «nécessaire pour prononcer le texte, soit en le récitant, soit en le chantant» (art. 62). Enfin, les dernières sections de l'instruction (70 à 130) concernent les aspects d'ordre technique et juridique entourant la préparation des traductions, la demande de recognitio (approbation) pour garantir leur authenticité, la composition des commissions chargées des traductions et la publication des livres liturgiques.

La traduction de la Bible et des documents liturgiques selon le Vatican requiert des qualités et des compétences très particulières. On exige beaucoup du traducteur. Outre la connaissance de l'hébreu, du grec et du latin, il lui faut avoir « un esprit de prière et la confiance en l'aide de Dieu» (art. 75); être profondément croyant, docile et soumis au Magistère de l'Église; avoir la conviction que les textes sacrés ont été composés sous l'inspiration divine et qu'ils cachent la révélation du mystère du salut; adhérer pleinement aux dogmes catholiques; croire aux miracles; accepter de traduire littéralement certains passages sans les comprendre, car «les paroles de la Sainte Écriture [...] expriment des vérités qui dépassent les limites imposées par le temps et le lieu» (art. 19); croire que les obscurités dans les textes sont voulues par Dieu (art. 41); être conscient de contribuer à la propagation de la doctrine catholique; traduire le plus littéralement possible et en fonction de la tradition multiséculaire de l'Église; accepter que son travail soit révisé (art. 75) par des personnes versées dans les questions de doctrine, mais qui ne connaissent pas forcément les arcanes de la traduction.

Avant d'être admis dans une commission, les traducteurs devront montrer patte blanche et obtenir au préalable le Nihil obstat de la Congrégation pour le culte divin et la discipline des sacrements, qui considèrera leur formation, leur compétence et la lettre de recommandation de leur évêque diocésain. Qui plus est, ils devront accepter d'accomplir leur travail confidentiellement et dans le plus strict anonymat. Il semble bien révolu le temps où l'on désignait les Bibles du nom de leur traducteur: Bible de Wyclif, de Luther, de Tyndale, d'Olivétan, de Crampon, de Chouraqui, etc. La traduction n'étant pas perçue comme un acte de création, contrairement à l'opinion admise dans les milieux traductologiques, le traducteur doit s'effacer devant le texte sacré. Comment d'ailleurs pourrait-il faire acte de présence dans un texte entièrement investi de la présence divine?... Il y a une certaine logique dans cette exigence d'anonymat pour ceux qui croient à la thèse de l'inspiration. La traduction sera donc collective et anonyme ou elle ne sera pas.

\section{Conclusion}

Depuis l'Antiquité, chaque tentative de nouvelle traduction de la Bible suscite des critiques. Même la Vulgate fut critiquée par saint Augustin qui mit en doute l'autorité de Jérôme. Pour l'Église catholique, les textes bibliques et liturgiques ne constituent pas des documents d'intérêt purement historique ou culturel. D'inspiration divine, ils renferment le «mystère du salut ». La nature très particulière de ce corpus doctrinal exigeait donc l'élaboration d'un ensemble de règles de traduction appropriées. Ces 
règles, qui témoignent de la nostalgie d'un texte unique, immuable et intemporel, n’ont pas été conçues en fonction de normes régissant la traduction de textes littéraires ou pragmatiques, ni à la lumière de la réflexion moderne en traductologie, tant s'en faut, mais en fonction de la mission traditionnelle de l'Église catholique et dans un souci évident de préserver intacte la doctrine, menacée par la multiplication de traductions jugées tendancieuses et non conformes à l'enseignement officiel de l'Église. Les compétences exigées des traducteurs sont de nature à refroidir l'ardeur des plus fervents adeptes du prosélytisme. Tout un chacun ne pourra pas s'improviser traducteur pour se faire ouvrier dans la vigne du Seigneur... Il faudra ni plus ni moins réunir les qualités d'un saint Jérôme pour se faire agréer comme traducteur par le Vatican.

Quelle conception de la traduction et du traducteur se dégage de cette cinquième instruction post-conciliaire? En plus de leurs dictionnaires et de leurs ressources informatiques, les traducteurs de textes religieux peuvent-ils réellement compter sur l'aide de l'Esprit Saint pour accomplir leur travail, comme l'ont été, croit-on, les auteurs des textes sacrés? Les deux cosignataires de l'instruction en ont la ferme conviction: "La traduction des livres liturgiques requiert [...] un esprit de prière et la confiance en l'aide de Dieu, qui n'est pas accordée seulement aux traducteurs, mais à l'Église elle-même, durant tout le processus qui conduit à l'approbation d'un texte stable et définitif» (art. 75; c'est nous qui soulignons). Le scepticisme est de mise... Toute doctrine religieuse entretient une croyance en l'existence d'êtres surnaturels, et les traducteurs des commissions de traduction créées par les évêques sont certainement animés d'une foi profonde qui les porte à croire sans doute à cette inspiration divine. Se pose alors le problème des erreurs qui émaillent les traductions des «traducteurs inspirés ». Même le vénéré saint Jérôme n'a-t-il pas commis plusieurs contresens dans sa Vulgate? Par exemple, lorsque Moïse descend du Sinaï, ne l'a-t-il pas affublé de cornes, alors qu'il aurait dû écrire que «la peau de son visage rayonnait» (Ex 34,29)? Jérôme n'était-il pas guidé par l'Esprit? Le grand réhabilitateur du travail des traducteurs que fut Didier Érasme (1467-1536) avait combattu en son temps cette idée, sans grand succès, semble-t-il, car ce mythe s'est maintenu jusqu'au $\mathrm{xx}^{\mathrm{e}}$ siècle sous la plume de papes, d'évêques et d'archevêques: "Pourquoi Jérôme aurait-il eu besoin d'enseigner la méthode de traduire les saintes lettres, écrit l'humaniste lucide, si le don en est accordé par inspiration divine? Allons-nous faire remonter nos erreurs au Saint-Esprit et dire qu'il en est l'auteur?» (Érasme 1967, t. I : 385-386). Érasme de Rotterdam est demeuré croyant jusqu'à la fin de sa vie, sans jamais abdiquer pour autant son jugement critique; il savait distinguer croyance, crédulité et mythe.

Ce qu'on demande en fait aux traducteurs ce n'est pas de réexprimer dans une langue moderne le sens purement conceptuel ou culturel des textes bibliques ou liturgiques, mais leur «sens doctrinal et théologique» (art. 50), leur sens religieux tel que l'enseigne l'Église catholique. C'est pourquoi, par exemple, seuls des croyants sont en mesure d'exprimer « une vraie notion théologique de causalité divine» (art. 54), là où des non-croyants seraient tentés d'employer des mots qui rendraient l'idée d'une aide purement extérieure ou profane. Il n'est pas donné à tout le monde de croire aux miracles et aux interventions divines dans les affaires de la vie courante... Quoi qu'il en soit, dorénavant, seuls des croyants convaincus et reconnus tels par la hiérarchie ecclésiastique pourront participer aux projets de traduction dont les évêques prendront l'initiative. 
Les règles qui composent l'instruction Liturgiam authenticam représentent l'orthodoxie catholique en matière de traduction. Ce «décalogue» destiné aux traducteurs repose sur un certain nombre de présupposés:

- les textes bibliques et doctrinaux sont d'inspiration divine;

- ils renferment une Vérité éternelle et immuable;

- l'Église a la mission de garder intact le message divin;

- elle est la seule compétente pour interpréter les textes de sa doctrine;

- elle a reçu la mission de les faire connaître à tous les peuples de la Terre;

- la traduction est un mal nécessaire;

- elle est dangereuse, car elle altère les textes;

- elle risque de falsifier la doctrine en pervertissant le sens des textes;

- les équivalences dynamiques de la traduction libre (créatrice) déforment le sens des textes;

- l’Église doit donc encadrer étroitement le travail des traducteurs;

- elle seule peut juger de la validité doctrinale d'une traduction;

- le traducteur à l'esprit critique est un hérétique en puissance;

- le traducteur croyant, docile et soumis au Magistère de l'Église peut, lui, compter sur l'aide de l'Esprit Saint;

- il est possible de traduire le sens exact et authentique des textes bibliques et doctrinaux;

- la traduction est une translatio, une forme de report non déformant;

- les obscurités des textes sont voulues par Dieu;

- il ne faut pas chercher à les éclaircir;

- la traduction littérale est la seule stratégie apte à rendre l'authenticité des textes;

- il existe une langue du religieux chrétien distincte du langage commun;

- il est impératif de respecter cette langue particulière;

- ce faisant, on maintient la doctrine, la tradition et la crédibilité de l'Église;

- il est plus important d'être fidèle à la tradition que de renouveler la langue du religieux chrétien;

- l'objet de la traduction est le sens doctrinal des textes;

- la traduction doit être une ouvre collective;

- le traducteur peut et doit être absent de ses traductions;

- seul un croyant convaincu peut traduire la Bible et les textes liturgiques comme l'entend l'Église catholique.

En y regardant de plus près, on constate que bon nombre de ces présupposés sont des dogmes (ou croyances institutionnalisées non fondées), des préjugés à l'égard des traducteurs, des idées reçues (fausses) sur la nature de la traduction, ou des mythes. Cette méthode idoine dénote dans l'ensemble une méconnaissance des véritables enjeux de la traduction ou, si l'on préfère, des enjeux de la véritable traduction. L'attachement obstiné que ses auteurs portent à la langue du religieux chrétien est contraire à l'essence même de la démarche de traduction qui est, pour une bonne part, réinvention dans la langue d'accueil et non mimétisme de la langue d'origine. «À trop vouloir défendre les formes figées du langage de la tradition, les mots de nos pères, nous n'inventons plus les conditions d'une transmission possible» (Boyer 2002: 124) du véritable message chrétien. Ce conservatisme est contre-productif.

Par ailleurs, bien que les auteurs de l'instruction insistent sur le fait que les traductions doivent être « exemptes de toute arrière-pensée idéologique» (art. 3, 32), il n'en demeure pas moins qu'ils demandent explicitement aux traducteurs de christianiser l'Ancien Testament en montrant que le Nouveau donne un sens à l'Ancien. Cela est 
en soi une distorsion idéologique. Dire, par exemple, dans Isaïe que c'est une «vierge» qui a enfanté pour faire d'avance allusion à la Vierge mère du Christ est abusif, car le texte original dit alma (une jeune femme) et non betoula (une vierge). De même, vox clamantis in deserto: "Une voix parle dans le désert / ouvrez un chemin au Seigneur» est une formulation qui fausse le sens réel de ce passage dont la signification exacte est: «Une voix appelle / dans le désert ouvrez le chemin » (Isaïe $40,3)^{26}$. Nous avons vu, également, que le choix des langues dans lesquelles pourront se faire les traductions est aussi d'ordre idéologique.

La cinquième instruction expose on ne peut plus clairement les nouvelles dispositions adoptées par l'Église en matière de traduction. Si l'encyclique de Pie XII et plus encore la Constitution conciliaire manifestaient une ouverture à l'égard des traductions, que l'on souhaitait adaptées aux fidèles d'aujourd'hui (traductionsinculturation), force est de constater, un demi-siècle plus tard, un retour du balancier du côté du traditionalisme et du conservatisme (traductions-tradition). Le littéralisme peut être vu comme une forme d'expression du conservatisme, voire un désir d'immobilisme, une idéalisation du passé. Dans Liturgiam authenticam, les qualificatifs « authentique», « exacte», «fidèle», «intégrale», «juste», "précise », «sûre» appliqués à la traduction sont autant de synonymes de l'expression «traduction-littéraleconforme-à-la-doctrine-et-à-la-tradition ». Rome a, semble-t-il, mis un frein au mouvement d'inculturation, ce que beaucoup de croyants actifs dans l'Église déplorent. Son nouveau traité de traduction porte l'empreinte de ce revirement. Ce traité conservateur bride les traducteurs et leur impose un ensemble de règles rigides. La créativité inhérente au processus de traduction est écrasée sous la chape de plomb du dogmatisme catholique et de la tradition. Faut-il s'en étonner quand on sait que le préfet de la Congrégation pour le culte divin et la discipline des sacrements, cosignataire de l'instruction, le cardinal Jorge A. Medina Estévez, d'origine chilienne, est un ultraconservateur? Personne ne contestera non plus que le pontificat de Jean-Paul II se caractérisait par un retour aux valeurs défendues bec et ongles par la droite catholique, attitude qui contraste singulièrement avec le vent de renouveau que Jean XXIII avait fait entrer dans l'Église en convoquant Vatican II. Cela prouve une fois de plus que la manière de traduire suit de près l'évolution des mentalités, des sociétés, des institutions et des idéologies. Les règles de la traduction religieuse ne font pas exception à cette loi universelle de l'art de traduire. Elles changent elles aussi au gré des circonstances, des réalités nouvelles, des virages idéologiques ou des courants de pensée qui dominent à un moment donné au sein d'une institution religieuse. La traduction sera toujours un bon baromètre du changement. De ce point de vue, elle ne trahit jamais.

\section{NOTES}

1. L'auteur tient à remercier Sybil Brake (Ottawa), Carmen Gloria Garbarini (Temuco, Chili), Paul Horguelin (Roxton Falls, Québec) et Christiane Lafond (Gatineau, Québec) pour avoir relu son texte et lui avoir fait part de leurs judicieux commentaires.

2. «Bible translation is a typical Christian activity.»

3. Ce n'est qu'au XIII ${ }^{\mathrm{e}}$ siècle que l'on a commencé à désigner la traduction latine de Jérôme sous le nom de «Vulgate», c'est-à-dire «texte commun», «texte populaire».

4. En fait, en Angleterre deux siècles plus tôt, le théologien John Wyclif (v.1320-1384), en prônant la séparation de l'Église et de l'État, en s'attaquant au pape et en faisant de la Bible la base de la foi, fait figure de précurseur de la Réforme. On l'a d'ailleurs surnommé le «premier des protestants». Sa 
traduction de la Bible publiée en 1382 est probablement la plus importante œuvre traduite en anglais au XIV ${ }^{\mathrm{e}}$ siècle.

5. «La Réforme [n'a-t-elle] pas été avant tout une querelle de traducteurs?» (Cary 1963: 7).

6. On appelle «édition typique» un livre publié en vertu d'un décret de la Congrégation pour le culte divin et la discipline des sacrements. Les éditions typiques publiées avant la cinquième instruction (2001) étaient diffusées par l'Imprimerie Polyglotte Vaticane ou par la Libreria Editrice Vaticana; à l'avenir, elles seront normalement imprimées par l'Imprimerie vaticane et leur diffusion sera réservée de droit à la Libreria Editrice Vaticana (Liturgiam authenticam, 2001, art. 109).

7. Voir plus haut l'opinion contraire exprimée par ses prédécesseurs.

8. Ses prédécesseurs, Pie X et Pie XI, ne s'étaient pas opposés à la révision de la version latine de la Vulgate, mais sans aller jusqu'à recommander sa traduction en d'autres langues.

9. Strictement parlant, on ne peut guère parler de "textes originaux » dans le cas des textes bibliques, car seules des copies des textes primitifs sont parvenues jusqu'à nous. Les textes les plus traduits au monde demeurent toujours sans originaux.

10. L'auteur de cette encyclique est conscient que l'opération de traduction exige du traducteur qu'il connaisse «le caractère particulier de l'écrivain sacré et ses conditions de vie, l'époque à laquelle il a vécu, les sources écrites ou orales qu'il a employées [et] sa manière d'écrire» (art. 34), afin de mieux connaître le sens réel de ce que l'hagiographe a voulu exprimer. C'est là un point de vue très moderne. Les théoriciens actuels prônent aussi la connaissance des compléments cognitifs : auteurs, style, contexte social, influences, etc.

11. On peut considérer que la traduction de l'Ancien Testament en grec, la Septante, réalisée au III ${ }^{\mathrm{e}}$ siècle av. J.-C., est, d'un point de vue historique, la première forme d'inculturation subie par les textes bibliques.

12. Cette commission est présidée par l'évêque de Fulda (Allemagne), Edward Schick.

13. La preuve en est que cette editio typica connaîtra pas moins de quatre rééditions et révisions entre 1984 et 1992. Comme quoi il n'y a jamais de traduction définitive. Compagnons d'infortune de Sisyphe, les traducteurs sont condamnés à réviser sans fin leurs propres traductions et à refaire celles de leurs prédécesseurs.

14. ... ou, au Québec, en «joual», comme il s'en est publié dans les années 1990. Voir à ce propos «L'Évangile selon Ti-Marc!» (Anonyme 1991), «Le mépris du peuple» (Gagnon 1991) et «La Bible “traduite” ou l'abrutissement à la québécoise» (Martin 1991).

15. Peu de femmes ont traduit la Bible. Au XIx ${ }^{\mathrm{e}}$ siècle, l'Américaine Julia E. Smith (1792-1886) est, à notre connaissance, l'unique femme à l'avoir fait, seule, à partir du grec et de l'hébreu. Sa traduction très littérale, Holy Bible Containing the Old and New Testaments; Translated Literally from the Original Tongues, fut publiée en 1876. Sa compatriote Elizabeth Cady Stanton (1815-1902), fougueuse activiste, publia en 1895, sous le titre The Woman's Bible, une relecture féministe des passages de la Bible faisant référence aux femmes. Voir Von Flotow 2002.

16. Samuel Butler (1835-1902) avait exprimé une opinion similaire, lui qui fait dire à l'un de ses personnages: "Je traduirai tout avec cette liberté sans laquelle aucune traduction ne s'élève au-dessus du mot à mot» (Butler 1924: 56. Traduction: Valery Larbaud).

17. Cela s'est produit récemment. La version anglaise réalisée à grands frais pour le compte d'évêques de onze pays anglophones dans les années 1980 et soumise pour approbation en 1996, fut rejetée par le Vatican qui a estimé que cette traduction ne transmettait pas fidèlement certains points importants de la doctrine catholique. Roma locuta, causa finita (L'avis de Rome prévaut toujours).

18. Cosignée par le cardinal Jorge A. Medina Estévez, préfet de la Congrégation pour le culte divin et la discipline des sacrements, et par l'archevêque secrétaire, Francesco Pio Tamburrino.

19. «Élément de célébration qui est propre à un saint, un temps, un lieu, et ne fait partie ni de l'ordinaire ni du commun» (Le Petit Robert, 2000).

20. Dans notre manuel d'initiation à la traduction, La Traduction raisonnée (Delisle 2003: 165-172), nous utilisons ce terme pour décrire le premier des trois niveaux d'interprétation de l'opération traduisante, les deux autres étant la «remémoration» et la «création discursive». Le report est l'«opération du processus de la traduction par laquelle certains éléments d'information du texte de départ qui ne nécessitent pas une analyse interprétative sont transcodés tels quels ou non dans le texte d'arrivée» (Delisle, Cormier, Lee-Jahnke 1999: 68).

21. L'auteur du Génie du christianisme ne cache pas sa prédilection pour le littéralisme dans son «Essai sur la littérature angloise » : «La traduction littérale me paraît toujours la meilleure: une traduction interlinéaire serait la perfection du genre, si on lui pouvait ôter ce qu'elle a de sauvage» (Chateaubriand $1837: 4$ ), et dans ses «Remarques» qui précèdent sa traduction du Paradis perdu de Milton: 
«C'est une traduction littérale dans toute la force du terme que j'ai entreprise, une traduction qu'un enfant et un poète pourront suivre sur le texte, ligne à ligne, mot à mot, comme un dictionnaire ouvert sous leurs yeux» (Chateaubriand 1871 : ix); «J'ai calqué le poème de Milton à la vitre, peutêtre en résultera-t-il cette conviction que la version littérale est ce qu'il y a de mieux pour faire connaître un auteur tel que Milton» (ibid. : xi).

22. "I am somewhat of a "literalist", because I am for truth and accuracy" (Newmark 1988: ix). " [...] we do translate words because there is nothing else to translate; there are only the words on the page; there is nothing else there» (ibid.: 73).

23. C'est le cas notamment de l'Association des Traducteurs Littéraires de France (ATLF): «Le traducteur s'abstient de traduire une œuvre à partir d'une autre traduction en langue étrangère, dite traduction-relais, à moins que l'auteur ne l'y autorise» (art. 3).

24. «Livre dans lequel se trouvent les textes de la Bible (lectionnaire proprement dit) ou des Pères (lectionnaire patristique, ou autrefois sermonnaire ou homéliaire) qui doivent être lus à la messe et à l'office» (Le Grand dictionnaire terminologique de l'Office québécois de la langue française <http:// www.oqlf.gouv.qc.ca/ressources/gdt.html>).

25. Bible des communautés juives d’Égypte, elle fut aussi la Bible des pères de l'Église et la seule Bible des chrétiens pendant des siècles (Cousin 1990: 5).

26. Ces deux exemples sont empruntés à Henri Meschonnic (2001: 13; $2004: 157)$.

\section{RÉFÉRENCES}

Anonyme (1991) : «L'Évangile selon Ti-Marc!», dans L'Actualité, $1^{\text {er }}$ juin, p. 11 (Reproduit sur le cédérom Delisle et Lafond 2005).

Association des Traducteurs Littéraires de France (ATLF): "Code de déontologie du traducteur littéraire» (<www.atlf.org/association/deontologie.html>, site consulté le 15-11-2004).

Berman, A. (1995) : Pour une critique des traductions: John Donne, Paris, Gallimard, coll. «NRF».

Berner, C. (1999): «Le penchant à traduire», dans F. Schleiermacher, Des différentes méthodes du traduire, Paris, Éditions du Seuil, coll. «Points. Essais», n 402, p. 11-26.

Boyer, F. (2001) : La Bible, trad. sous la dir. de Frédéric Boyer, Jean-Pierre Prévost et Marc Sevin, Paris, Bayard / Montréal, Médiaspaul.

Boyer, F. (2002) : La Bible, notre exil, Paris, P.O.L.

Butler, S. (1924): Nouveaux voyages en Erewhon accomplis, vingt ans après la découverte du pays, par le premier explorateur et par son fils, trad. par Valery Larbaud, $4^{\text {e }}$ éd., Paris, Éditions de la Nouvelle Revue française.

CARY, E. (1963): Les Grands traducteurs français, Genève, Librairie de l'Université.

Chateaubriand, Fr. R. de (1837): «Essai sur la littérature angloise. Avertissement», dans CEuvres complètes, Paris, Pourrat Frères, t. XXIII, p. 3-15 (Reproduit sur le cédérom Delisle et Lafond 2005).

Chateaubriand, Fr. R. de (1871): «Remarques», dans Le Paradis perdu, Paris, Garnier Frères, xx-579 (Reproduit sur le cédérom Delisle et Lafond 2005).

Chouraqui, A. (1989): La Bible, Bruxelles, Desclée de Brouwer.

Chouraqui, A. (1990): L'amour fort comme la mort, Paris, Robert Laffont, coll. "Vécu».

Chouraqui, A. (2002) : "Traduire la Bible et le Coran au xx siècle», dans Circuit, nº 77, p. 6-9 (Reproduit sur le cédérom Delisle et Lafond 2005).

Claudel, P. (1966): Psaumes. Traductions 1918-1953, texte établi et annoté par Renée Nantet et Jacques Petit, Paris, Desclée de Brouwer.

Congrégation des rites (1964): Inter Ecumenici (<www.ceremoniaire.net/pastorale1950/docs/ interoecumenici.html>, site consulté le 28-12-2004) (Reproduit sur le cédérom Delisle et Lafond 2005).

Congrégation des rites (1967): Tres abhinc annos (<www.ceremoniaire.net/depuis1969/docs/liturgicae_ inst.html>, site consulté le 28-12-2004) (Reproduit sur le cédérom Delisle et Lafond 2005).

Congrégation pour le culte divin (1970): Liturgicae instaurationes, (<www.ceremoniaire.net/depuis1969/ docs/liturgicae_inst.html $>$, site consulté le 28-12-2004) (Reproduit sur le cédérom Delisle et Lafond 2005).

Congrégation pour le culte divin et la discipline des sacrements (1994): Varietates legitimae, (<www. adoremus.org/doc_inculturation.html>, site consulté le 28-12-2004) (Reproduit sur le cédérom Delisle et Lafond 2005).

Congrégation pour le culte divin et la discipline des sacrements (2001): Liturgiam authenticam, (<www. vatican.va/roman_curia/congregations/ccdds/documents/rc_con_ccdds_doc_20010507_liturgiamauthenticam_fr.html>, site consulté le 28-12-2004) (Reproduit sur le cédérom Delisle et Lafond 2005) 
Cousin, H. (dir.) (1990) : «La Bible grecque. La Septante», dans Service biblique évangile et vie, Cahiers Évangile, Paris, Éditions du Cerf, supplément au n ${ }^{\circ} 74$.

Delisle, J. (1990): «Le froment du sens, la paille des mots», dans Études traductologiques en hommage à Danica Seleskovitch, textes réunis par Marianne Lederer, Paris, Minard, coll. "Lettres modernes», p. 61-72.

Delisle, J. et J. Woodsworth (dir.) (1995): Les Traducteurs dans l'histoire, Ottawa, Les Presses de l'Université d'Ottawa / Paris, Les éditions UNESCO, coll. «Pédagogie de la traduction », nº 2, publié sous les auspices de la Fédération internationale des traducteurs.

Delisle, J., M. C. Cormier et H. Lee-Jahnke (dir.) (1999): Terminologie de la traduction / Translation terminology / Terminología de la traducción / Terminologie der Übersetzung, Amsterdam, John Benjamins, coll. «FIT Monograph / Collection FIT», $n^{\circ} 1$.

Delisle, J. (2001): Compte rendu de Gloires. Traduction des psaumes par H. Meschonnic, dans TTR, vol. 14, n 1, p. 235-245 (Reproduit sur le cédérom Delisle et Lafond 2005).

Delisle, J. (2003): La Traduction raisonnée, $2^{\mathrm{e}}$ éd., Ottawa, Les Presses de l'Université d'Ottawa, coll. «Pédagogie de la traduction».

Delisle, J. et G. LAfond (2005): Histoire de la traduction (cédérom pour PC), Gatineau (Québec), édition restreinte aux seules fins d'enseignement par J. Delisle, professeur, École de traduction et d'interprétation, Université d'Ottawa.

Denzinger, H. (2001) : Titre Symboles et définitions de la foi catholique, édité par Peter Hünermann pour l'édition originale et par Joseph Hoffmann pour l'édition française, Paris, Éditions du Cerf, 2001, LI1283 p. (<www.jesusmarie.com/denzinger.html>, site consulté le 28-12-2004)

Érasme, D. (1967) : «Lettre à Christophe Fisher, Paris, vers mars 1505 », dans La correspondance d'Érasme, traduite et annotée sous la dir. d'Aloïs Gerlo et de Paul Foriers d'après l'Opus epistolarum de P. S. Allen, H. M. Allen et H. W. Garrod, Bruxelles, Presses académiques européennes, 1967, t. 1, p. 385386 (Reproduit sur le cédérom Delisle et Lafond 2005).

Gagnon, L. (1991): «Le mépris du peuple», dans La Presse, 15 juin, p. B-3 (Reproduit sur le cédérom Delisle et Lafond 2005).

GrégoIre XVI (1844): Inter praecipuas machinationes, lettre encyclique contre les sociétés bibliques (www.jesusmarie.com/denzinger.html, site consulté le 28-12-2004).

Ivereigh, A. (2004) : «The Battle over the Translations of the Catholic Mass», dans Translating Today, $\mathrm{n}^{\circ} 1, \mathrm{p} .14-15$.

Kelly, J. N. D. (1975) : Jerome: His Life, Writings, and Controversies, Londres, Duckworth.

Kelly, Louis G. (1976) : To Pammachius: On the Best Method of Translating (St. Jerome Letter 57), traduite et annotée par L. G. Kelly, Université d'Ottawa, École de traducteurs et d'interprètes, coll. « Working Papers in Translatology», $\mathrm{n}^{\circ}$ 1, 26 p. (Reproduit sur le cédérom Delisle et Lafond 2005).

Martin, S. (1991): «La Bible "traduite" ou l'abrutissement à la québécoise», dans Le Devoir, 19 juin, p. B-8 (Reproduit sur le cédérom Delisle et Lafond 2005).

Medina Estévez, J. A. (2001) : "Cardinal Estévez' Response to Criticism of Liturgiam Authenticam», dans Notitiae, vol. 37 (Reproduit sur le site <www.bible-researcher.com/liturgiam-authenticam3.html>, consulté le 6 janvier 2005).

Meschonnic, H. (2001): Gloires. Traduction des psaumes, Paris, Desclée de Brouwer.

Meschonnic, H. (2004): Un coup de Bible dans la philosophie, Paris, Bayard.

Naвokov, V. (1955) : «Problems of Translation: Onegin in English», dans Partisan Review, vol. 22, $\mathrm{n}^{\circ} 4$, p. $496-512$.

Newmark, P. (1988): A Textbook of Translation, Londres / New York, Prentice Hall.

PAul VI (1963): Sacrosanctum Concilium, Constitution sur la sainte liturgie (<www.vatican.va/archive/ hist_councils/ii_vatican_council/documents/vat-ii_const_19631204_sacrosanctum-concilium_fr. html>, site consulté le 28-12-2004) (Reproduit sur le cédérom Delisle et Lafond 2005).

PIE VII (1816): "Lettre Magno et acerbo à l'archevêque de Moghilev», 3 septembre 1816 (<www. jesusmarie.com/denzinger.html>, site consulté le 28-12-2004).

PIE IX (1846): Qui pluribus, lettre encyclique sur la foi et la religion (membres.lycos.fr/lesbonstextes/ pixquipluribus.htm, site consulté le 28-12-2004)

PIE XII (1943): Divino Afflante Spiritu, lettre encyclique sur les études bibliques (<www.vatican.va/ holy_father/pius_xii/encyclicals/documents/hf_p-xii_enc_30091943_divino-afflante-spiritu_fr. html>, site consulté le 28-12-2004) (Reproduit sur le cédérom Delisle et Lafond 2005)

Pyм, A. (1997) : Pour une éthique du traducteur, Ottawa, Les Presses de l'Université d'Ottawa, coll. « Regards sur la traduction». 
Rabin, C. (1972): "Cultural Aspects of Bible Translations», dans Babel, vol. XVIII, n 3, p. 11-20. (Reproduit sur le cédérom Delisle et Lafond 2005)

Renken, A. (2002) : La Représentation de l'étranger. Une réflexion herméneutique sur la notion de traduction, Lausanne, Université de Lausanne, Cahiers du Centre de Traduction Littéraire, $\mathrm{n}^{\circ} 42$.

Renouveau de la liturgie pastorale en France après 1950 (<www.ceremoniaire.net/pastorale1950/ index.html>, site consulté le 28-12-2004)

Shorter, A. «Inculturation of African Traditional Religious Values in Christianity-How Far?» (<www. afrikaworld.net/afrel/shorter.htm>, site consulté le 31-12-2004) (Reproduit sur le cédérom Delisle et Lafond 2005)

Von Flotow, L. (2002): «Julia E. Smith, traductrice de la Bible, à la recherche de la vérité par le littéralisme», dans Jean Delisle (dir.), Portraits de traductrices, Ottawa, Les Presses de l'Université d'Ottawa / Arras, Artois Presses Université, p. 291-319. 